\title{
EFECTOS A CORTO PLAZO DE LA CONTAMINACIÓN ATMOSFÉRICA SOBRE LA MORTALIDAD. RESULTADOS DEL PROYECTO EMECAM EN VITORIA-GASTEIZ, 1990-94 *
}

\author{
María José Pérez Boillos (1), Andrés Alonso López (2) Juan José Estibalez González (1) y Miguel \\ Ángel García Calabuig (3)
}

(1) Departamento Municipal de Salud y Consumo del Ayuntamiento de Vitoria-Gasteiz.

(2) Servicio de Medio Ambiente del Ayuntamiento de Vitoria-Gasteiz.

(3) Departamento de Sanidad del Gobierno Vasco.

(*) Este trabajo cuenta con una beca del Fondo de Investigaciones Sanitarias (Expediente núm 97/0051-10).

\section{RESUMEN}

Fundamento: El objetivo de este trabajo es evaluar la asociación a corto plazo entre los niveles de humos negros $(\mathrm{HN})$ y dióxido de azufre $\left(\mathrm{SO}_{2}\right)$ y la mortalidad en Vitoria-Gasteiz para un período de cinco años, mediante la aplicación de la metodologia de análisis estandarizada del proyecto EMECAM.

Métodos: Estudio ecológico de series temporales que trata de estimar la asociación entre las variaciones diarias de mortalidad (mortalidad total en todas las edades y mortalidad total en mayores de 70 años), y contaminación atmosférica (dióxido de azufre- $\mathrm{SO}_{2}$ y humos negros), utilizando modelos de regresión de Poisson. Se siguió la metodología EMECAM.

Resultados: La mediana de muertes diarias fue de tres en el total de la población y de dos en ancianos. El nivel medio de humos negros fue $51,15 \mu \mathrm{g} / \mathrm{m}^{3}$ y el de $\mathrm{SO}_{2} 18,04 \mu \mathrm{g} / \mathrm{m}^{3}$. Se encontró una asociación estadísticamente significativa entre humos negros y mortalidad en ancianos durante el semestre frío, con un RR de 1,014 (IC 95\%: 1,002-1,026), correspondiente a un incremento de $10 \mu \mathrm{g} / \mathrm{m}^{3}$ del contaminante. Parecía detectarse un umbral en $80-90 \mu \mathrm{g} / \mathrm{m}^{3}$ de humos negros. La asociación con $\mathrm{SO}_{2}$ no fue significativa.

Conclusiones: Los niveles de humos negros del período es tudiado se asocian a un incremento en la mortalidad en ancianos, coincidiendo con el resultado de otros estudios.

Palabras clave: Mortalidad. Contaminación atmosférica. Series temporales. Proyecto EMECAM. Regresión de Poisson. Partículas. Humos negros. Dióxido de azufre. Contaminantes ambientales.

\section{ABSTRACT \\ Short-term Effects of Air Pollution on the Mortality. Results of the $E M E C A M$ Project in the city of Vitoria-Gasteiz, 1990-1994}

Background: The objective of this article is that of assessing the short-term relationship between the balck smoke (SM) and $\mathrm{SO}_{2}$ levels and the mortality in Vitoria-Gasteiz over a five-year period by means of employing the procedure for analysis standardized in the EMECAM Project.

Methods: Ecological time series study aimed at estimating the relationship between the daily fluctuations in the mortality (total mortality of all ages and total death rate for those over age 70 ) and air pollution (sulfur dioxide- $\mathrm{SO}_{2}$ and black smoke), employing the Poisson regression models. The EMECAM methodology was followed.

Results: The median of daily deaths was three for the entire population and two for the elderly. The mean black smoke level was $51.15 \mu \mathrm{g} / \mathrm{m}^{3}$ and that of $\mathrm{SO}_{2} 18.04 \mu \mathrm{g} / \mathrm{m}^{3}$. A statistically significant relationship was found to exist between black smoke and the mortality for the elderly through the cold half of the year, with an RR of 1.014 ( $\left.\mathrm{Cl}_{95 \%}: 1.002-1.026\right)$, pertinent to a $10 \mu \mathrm{g} / \mathrm{m}^{3}$ rise in the pollutant. A threshold at $80-90 \mu \mathrm{g} / \mathrm{m}^{3}$ seemed to be detected for black smoke. The relationship with $\mathrm{SO}_{2}$ was not significant.

Conclusions: The black smoke levels for the period studied are related to a rise in the mortality among the elderly, tallying with the results of other studies.

Key words: Mortality. Air pollution. Time series. EMECAM Project. Poisson regression. Particles. Black smoke. Sulfur dioxide. Environmental pollutants.

Correspondencia:

Andrés Alonso López

Servicio de Medio Ambiente

Ayuntamiento de Vitoria-Gasteiz

San Prudencio, 30

01005 Vitoria-Gasteiz 


\section{INTRODUCCIÓN}

En Vitoria-Gasteiz no existen trabajos previos que hayan cuantificado el efecto de la contaminación atmosférica sobre la salud. La ciudad cuenta con datos de monitorización de dióxido de azufre $\left(\mathrm{SO}_{2}\right)$ y partículas en suspensión (humos negros) desde 1984 y no ha registrado episodios importantes de contaminación. Las principales fuentes de contaminación son el tráfico y la industria. Los niveles de humos negros detectados habitualmente son moderados y los de $\mathrm{SO}_{2}$ bajos, inferiores a los registrados en la mayoría de las ciudades europeas participantes en otros estudios que han valorado la relación entre contaminación atmosférica y mortali$\mathrm{dad}^{1-10}$.

El objetivo de este trabajo es evaluar la asociación a corto plazo entre los niveles de humos negros $(\mathrm{HN})$ y $\mathrm{SO}_{2}$ y la mortalidad en Vitoria-Gasteiz para un período de cinco años, mediante la aplicación de la metodología de análisis estandarizada del proyecto EMECAM $^{11,12}$.

\section{MATERIAL Y METODO}

Se realizó un estudio ecológico de series temporales que utilizó el día como unidad de análisis, y con él se pretende estimar la asociación entre las variaciones diarias de mortalidad y contaminación atmosférica mediante la construcción de diferentes modelos de regresión de Poisson. Tanto en la elección de las variables como en la construcción del modelo, se siguió el procedimiento EMECAM, ya presentado con detalle en otros artículos de este número ${ }^{11,12}$.

\section{Mortalidad}

Como variables respuesta se utilizaron el número de defunciones diarias en todas las edades, por todas las causas excluyendo las externas (CIE-9 001-799) y el número de defunciones diarias por todas las causas, ex- cluyendo las externas, en personas de $70 \mathrm{o}$ más años. Los datos fueron facilitados por el EUSTAT (Instituto Vasco de Estadística) y el Departamento de Sanidad del Gobierno Vasco. Las series incluían las muertes de personas residentes en Vitoria-Gasteiz sucedidas desde el 1 de Enero de 1990 al 31 de Diciembre de 1994.

\section{Contaminación}

La información sobre la contaminación atmosférica se obtuvo de la Red de Vigilancia gestionada por el Ayuntamiento de Vitoria-Gasteiz. La ciudad cuenta con una red manual que mide los valores medios de 24 horas de $\mathrm{SO}_{2}$ y humos negros. La toma de muestras de los equipos está situada aproximadamente a tres metros del suelo. Los datos son recogidos desde las 0 horas de un día hasta las 0 horas del día siguiente. La medición de las partículas en suspensión se realiza por el método del humo normalizado, con retención de las partículas en un filtro tipo Whatman $n .{ }^{\circ} 1$ y cuantificación mediante un método reflectométrico (reflectómetro EEL). Este método determina la intensidad de la negrura producida principalmente por partículas con un diámetro menor de $4 \mu \mathrm{m}^{13,14}$. La medición del $\mathrm{SO}_{2}$ se realizó, en 1990 por el método colorimétrico de la Thorina y en el resto del período de estudio por la captación del contaminante con $\mathrm{H}_{2} \mathrm{O}_{2}$ al $0,3 \%$ y la determinación de la concentración de anión sulfato por cromatografia iónica con supresión química para $\mathrm{SO}_{2}$. Antes de aceptar el cambio de técnica se realizó un estudio de tiabilidad entre ambos métodos, comprobándose que el índice de correlación era del 0,99 y que la diferencia de medias se situaba en torno a $1 \mu \mathrm{g} / \mathrm{m}^{3}$. Durante el período de estudio existían en la ciudad cuatro captadores manuales, situados en el área urbana, en áreas de tráfico medio e intenso. En cl estudio sólo se incluyeron tres de ellos, ya que el cuarto, mostraba un porcentaje de valores válidos inferior al 75\%; en los otros tres, este porcentaje estaba entre el 88,3-92,4\%. La correlación entre la estación descartada y el res- 
to era 0,7 . Entre las estaciones elegidas el índice de correlación era del $0,57-0,59$ para $\mathrm{HN}$ y 0,49 a 0,59 para $\mathrm{SO}_{2}$.

\section{Variables meteorológicas}

Las variables utilizadas fueron la temperatura media (media de la temperatura mínima y máxima de cada día) y la humedad relativa diaria (media de la humedad a las 0,7 , 13 y 18 horas). Ambas variables se obtuvieron de la información recogida por el Instituto Nacional de Meteorología en la estación del Aeropuerto de Foronda, a $5 \mathrm{Km}$ de la ciudad. Aunque existían otras estaciones dentro de la ciudad, se decidió elegir ésta porque los datos estaban disponibles de forma regular para todo el período de estudio.

\section{Gripe}

Los datos de gripe se obtuvieron a partir de las declaraciones semanales (EDOS) comunicadas al Servicio de Epidemiología de la Delegación Territorial de Sanidad de Alava. La cobertura media de declaración fue del $83,1 \%$, no variando de modo significativo a lo largo del período de estudio.

\section{RESULTADOS}

La mediana de muertes diarias a lo largo de todo el periodo fue de 3 para todas las edades (media de 3,5 ) y de 2 para el grupo de 70 o más años (media de 2,4). La serie presentó una distribución estacional aumentando el número de fallecimientos en el semestre frío respecto al cálido $(\mathrm{p}<0,01)$ (tabla 1$)$. No se regis-

Tabla 1

Contaminación, características meteorológicas, gripe, y mortalidad en Vitoria-Gasteiz (1990-94)

\begin{tabular}{|c|c|c|c|c|c|c|}
\hline & Periodo & media & $p 50$ & $p 10$ & $p 90$ & $\%$ perdidos \\
\hline \multirow[t]{3}{*}{ Humos negros $\left(\mu \mathrm{g} / \mathrm{m}^{3}\right)$} & todo el año & 51,15 & 45,00 & 19,33 & 88,33 & 1,6 \\
\hline & semestre frio & 57,95 & 49,67 & 21,33 & 102,67 & 2,9 \\
\hline & semestre cálido & 44,63 & 41,00 & 18,00 & 76,50 & 0,3 \\
\hline \multirow[t]{3}{*}{$\mathrm{SO}_{2}\left(\mu \mathrm{g} / \mathrm{m}^{3}\right)$} & todo el año & 18,04 & 16,00 & 7,33 & 31,33 & 1,8 \\
\hline & semestre frío & 22,31 & 20,33 & 8,33 & 38,00 & 2,9 \\
\hline & semestre cálido & 13,92 & 13,67 & 7,00 & 21,33 & 0,7 \\
\hline \multirow[t]{3}{*}{ Temperatura $\left({ }^{\circ} \mathrm{C}\right)$} & todo el año & 11,59 & 11,10 & 3,80 & 20,30 & 0 \\
\hline & semestre frío & 6,93 & 6,80 & 2,40 & 11,73 & 0 \\
\hline & semestre cálido & 16.18 & 16,00 & 10,30 & 22,00 & 0 \\
\hline \multirow[t]{3}{*}{ Humedad relativa $(\%)$} & todo el año & 76,47 & 77,00 & 61,00 & 90,00 & 0 \\
\hline & semestre frío & 80,20 & 82,00 & 67,00 & 92,00 & 0 \\
\hline & scmestrc cálido & 72,80 & 73,00 & 58,00 & 87,00 & 0 \\
\hline \multirow[t]{5}{*}{ Gripe (n. ${ }^{\circ}$ casos/día) } & todo el año & 66,49 & 36 & 10 & 130,90 & 0 \\
\hline & otoño & 54,86 & 55 & 27 & 83 & 0 \\
\hline & invierno & 158,94 & 112,0 & 43,4 & 315,00 & 0 \\
\hline & primavera & 39,94 & 30 & 16 & 79 & 0 \\
\hline & verano & 13,88 & 12 & 6 & 26 & 0 \\
\hline \multirow{3}{*}{$\begin{array}{l}\text { Muertes diarias en todos los } \\
\text { grupos de edad }\end{array}$} & todo el año & 3,53 & 3,00 & 1,00 & 6,00 & 0 \\
\hline & semestre frío & 3,82 & 4,00 & 1,00 & 6,00 & 0 \\
\hline & semestre cálido & 3,25 & 3,00 & 1,00 & 6,00 & 0 \\
\hline \multirow{3}{*}{$\begin{array}{l}\text { Muertes diarias en mayores } \\
\text { de } 70 \text { años }\end{array}$} & todo el año & 2,38 & 2,00 & 1,00 & 4,00 & 0 \\
\hline & semestre frío & 2,59 & 2,00 & 1,00 & 5,00 & 0 \\
\hline & semestre cálido & 2,17 & 2,00 & 0,00 & 4,00 & 0 \\
\hline
\end{tabular}


traron variaciones significativas en la mortalidad entre los años que componían el período de estudio, ni entre los días de la semana.

En cuanto a los contaminantes atmosféricos estudiados, los valores medios para todo el período fueron $51,15 \mu \mathrm{g} / \mathrm{m}^{3}$ para humos negros y $18,04 \mu \mathrm{g} / \mathrm{m}^{3}$ para $\mathrm{SO}_{2}$. En 52 de los días, los niveles medios diarios de $\mathrm{HN}$ superaron $\operatorname{los} 125 \mu \mathrm{g} / \mathrm{m}^{3}$; los de $\mathrm{SO}_{2}$ se mantuvieron siempre casi dos veces por debajo de ese valor (tabla 1). En los años estudiados se produjo una disminución en los valores de $\mathrm{HN}$, que pasaron de 61,7 a $43,82 \mu \mathrm{g} / \mathrm{m}^{3}(\mathrm{p}<0,01)$ y un ligero aumento en los de $\mathrm{SO}_{2}$ (de 15,4 a 21,2 $\left.\mu \mathrm{g} / \mathrm{m}^{3}, \mathrm{p}<0,01\right)$. Como era de esperar, los niveles medios de ambos contaminantes eran mayores en el semestre frío y disminuían durante los fines de semana. El índice de correlación entre los dos contaminantes fue 0,42 .

La temperatura media estuvo cercana a $\operatorname{los} 12^{\circ} \mathrm{C}$, con una diferencia aproximada de $10^{\circ} \mathrm{C}$ entre el semestre cálido y el frío. En cuanto a la humedad relativa, la media se situó en $76,5 \%$, siendo mayor en el semestre frío (tabla 1).
Tras la construcción de los modelos de regresión de Poisson para cada indicador de mortalidad, comprobamos que los residuos no presentaban autocorrelaciones significativas (figura 1-4).

La tabla 2 y las figuras 5 y 6 muestran los riesgos relativos, correspondientes a incrementos de $10 \mu \mathrm{g} / \mathrm{m}^{3}$ y a aumentos equivalentes al rango p90/p10 de cada contaminante, estimados para el global de la población y para el subgrupo con 70 o más años. Solamente resultó significativa la asociación entre humos negros y mortalidad en personas de 70 o más años durante el semestre frío del año. Un incremento de $10 \mu \mathrm{g} / \mathrm{m}^{3}$ de $\mathrm{HN}$ producía un aumento de $1,4 \%$ en el riesgo de morir. Cuando se valoró la relación entre este contaminante y la mortalidad en este grupo de edad durante todo el año, la asociación resultó débilmente significativa $(\mathrm{p}=0,053)$. En este caso el riesgo era ligeramente menor, ya que un aumento de $10 \mu \mathrm{g} / \mathrm{m}^{3}$ en el nivel de HN suponía una subida de $1,04 \%$ en el riesgo de morir. El resto de las asociaciones testadas mortalidad-contaminante no alcanzaron significación estadística, aunque fueron positivas en todas las épocas del año (exceptuando el caso de los $\mathrm{HN}$ durante el semestre cálido).

Tabla 2

Riesgos relativos de morir e intervalo de confianza al $95 \%$ (IC $95 \%$ ) asociados a incrementos de $10 \mu \mathrm{g} / \mathrm{m}^{3}$ de humos negros y dióxido de azufre $\left(\mathrm{SO}_{2}\right)$. Resultados para todo el año y por semestres. Vitoria-Gasteiz, 1990-94

\begin{tabular}{|lccccccc|}
\hline & \multicolumn{3}{c}{ Mortalidad en todas las edades } & & \multicolumn{2}{c|}{ Mortalidad en mayores 70 años } \\
\cline { 2 - 3 } \cline { 5 - 7 } & Ret & $R R$ & $I C 95 \%$ & Ret & $R R$ & $I C 95 \%$ \\
\hline Humos negros & & & & & & \\
Todo el año & 1 & 1,0063 & $0,9968-1,0160$ & 1 & 1,0104 & $0,9998-1,0210$ \\
Semestre frío & 2 & 1,0085 & $0,9979-1,0193$ & 1 & 1,0138 & $1,0017-1,0260$ \\
Semestre cálido & 4 & 0,9858 & $0,9678-1,0041$ & 4 & 0,9868 & $0,9665-1,0075$ \\
\hline SO 2 & & & & & & \\
Todo el año & 5 & 1,0204 & $0,9920-1,0496$ & 5 & 1,0237 & $0,9897-1,0588$ \\
Semestre frío & 5 & 1,0199 & $0,9890-1,0517$ & 5 & 1,0245 & $0,9873-1,0632$ \\
Semestre cálido & 2 & 1,0349 & $0,9678-1,1066$ & 0 & 1,0593 & $0,9781-1,1472$ \\
\hline
\end{tabular}

(Ret: retardo; RR: riesgo relativo) 
Figuras 1-4

Funciones de autocorrelación de los residıos de los modelos basales utilizados para cada indicador de mortalidad

\section{Mortalidad en todas las edades}

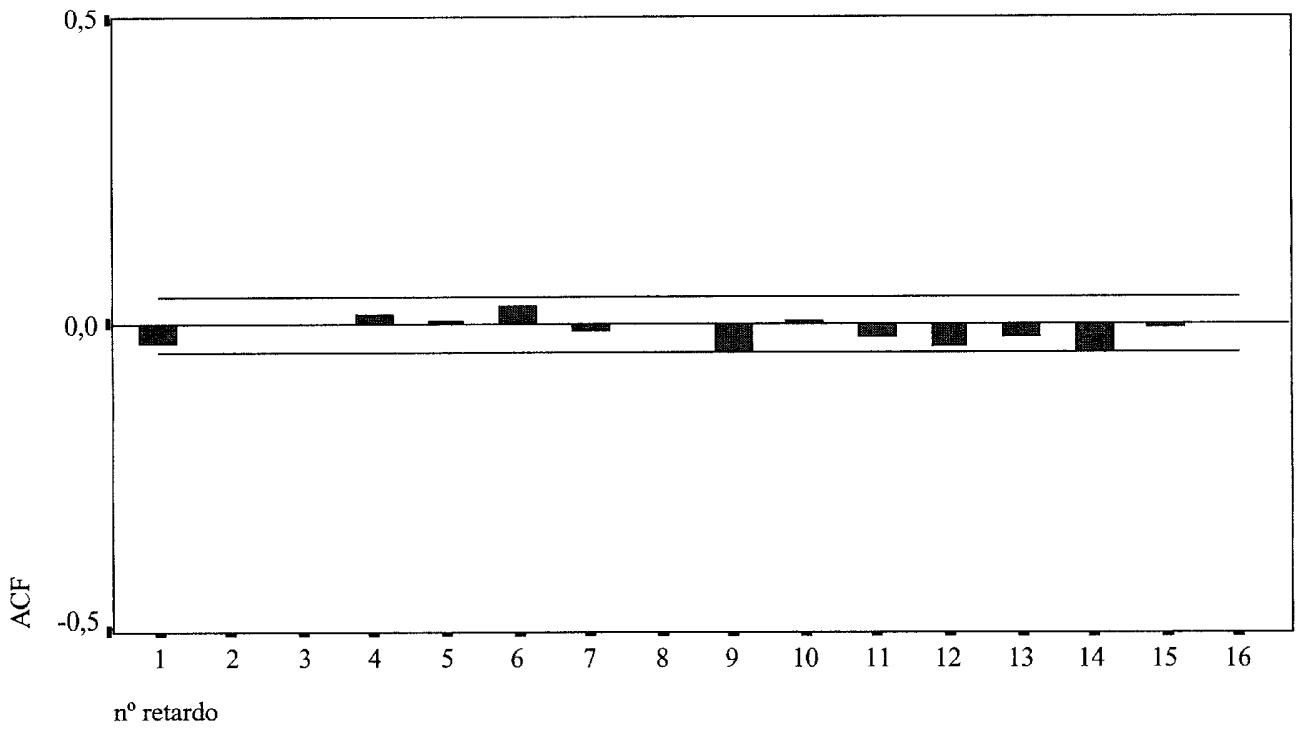

Mortalidad en 70 o más años

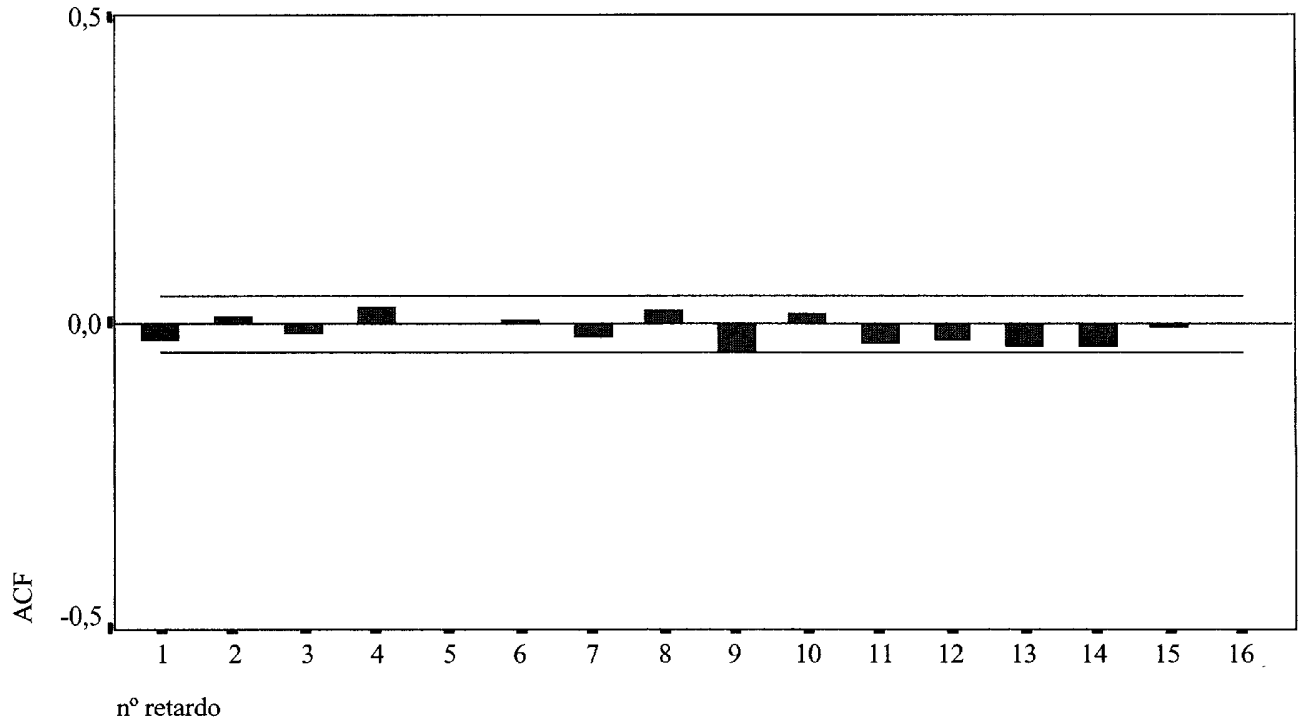


Figuras 1-4 (continuación)

Funciones de autocorrclación de los residuos de los modelos basales utilizados para cada indicador de mortalidad
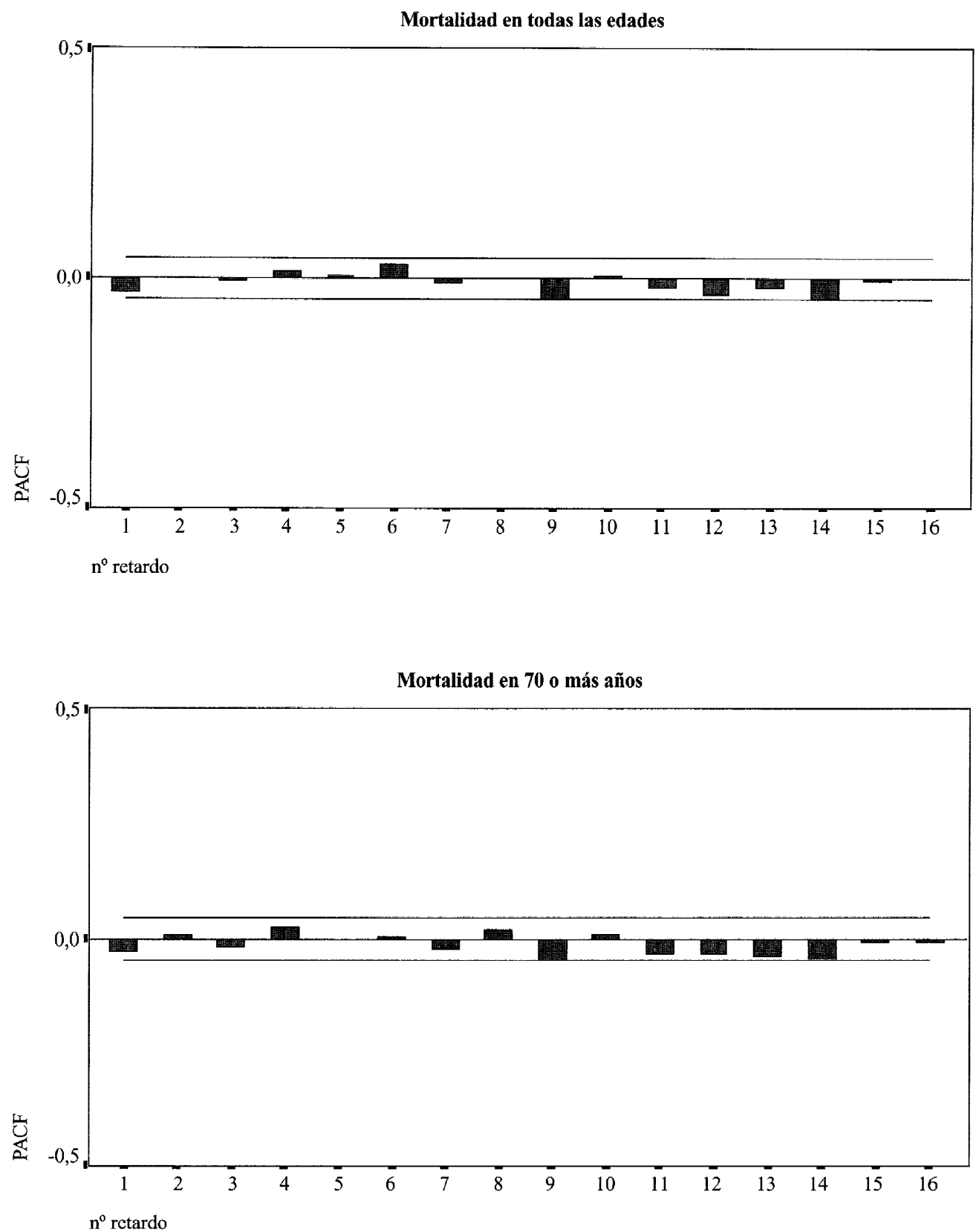
Figuras 5 y 6

Riesgos relativos de morir e intervalo de confianza al $95 \%$ (IC. $95 \%$ ) asociados a incrementos equivalentes al rango p90/p10 de humos negros y dióxido de azufre $\left(\mathrm{SO}_{2}\right)$. Resultados para todo el año y por semestres. Vitoria-Gasteiz, 1990-94

\section{Humos negros}

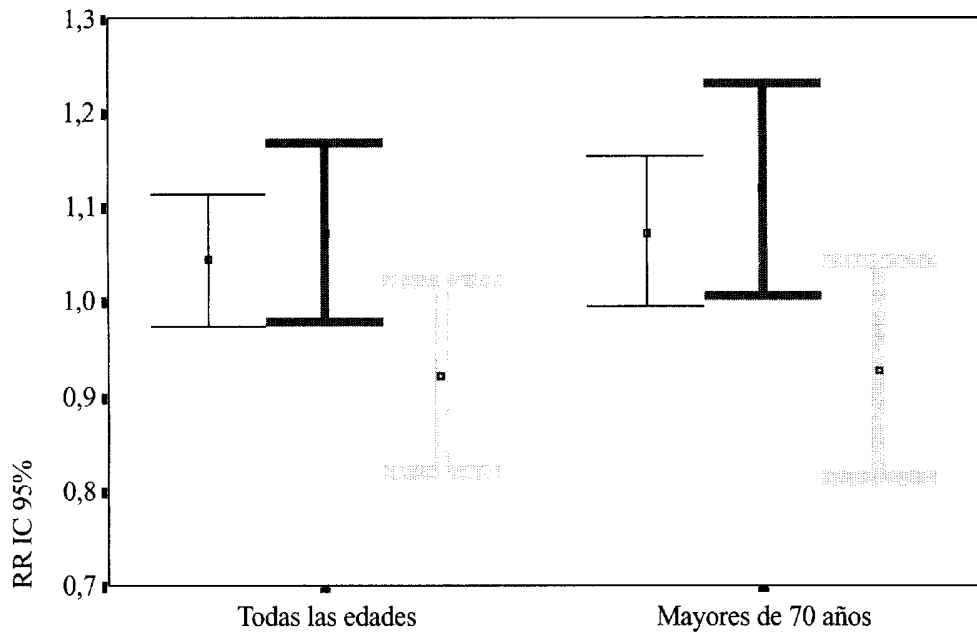

GRUPO DE EDAD

Dióxido de azufre

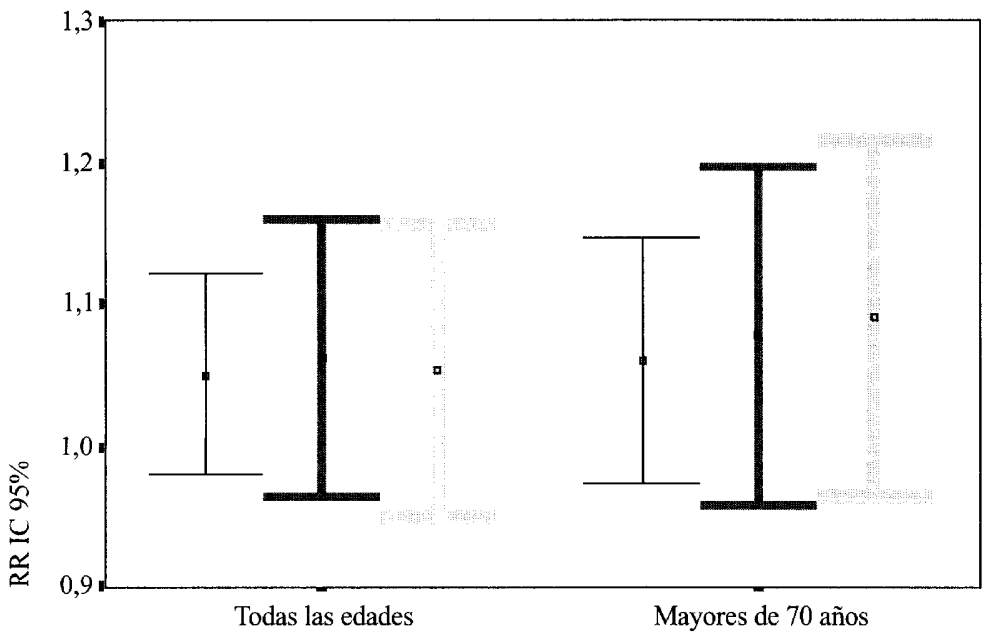

GRUPO DE EDAD

\section{SEMESTRE}

I RR global

RR semestre frío

- RR semestre cálido
SEMESTRE

I RR global

RR semestre frío

RR semestre cálido 
Las figuras 7 y 8 muestran la relación exposición-respuesta entre los residuos ajustados de mortalidad y los retardos de HN que resultaron más significativos en el análisis. En el caso de la mortalidad en personas de 70 o más años, la curva presentaba forma de meseta para los niveles más bajos del contaminante, y se hacía lineal a partir de $80-90$ $\mu \mathrm{g} / \mathrm{m}^{3}$ (cuando estudiábamos la mortalidad durante todo el año y el semestre frío) o a partir de $110-120 \mu \mathrm{g} / \mathrm{m}^{3}$ (cuando lo hacíamos para el semestre más cálido). Durante el semestre frío, la curva volvía a tener forma de meseta a partir de $120-130 \mu \mathrm{g} / \mathrm{m}^{3}$. Los diagramas de dispersión de $\mathrm{SO}_{2}$ no mostraban una clara relación dosis-respuesta.

\section{CONCLUSIONES}

A modo de conclusión, podemos decir que: 1) El estudio sugiere que existe una asociación positiva entre los niveles de contaminación atmosférica de Vitoria-Gasteiz durante el período de estudio y la mortalidad a corto plazo 2) La asociación más consistente entre los indicadores de mortalidad analizados y los contaminantes estudiados (HN y $\mathrm{SO}_{2}$ ) la encontramos con los humos negros, durante el semestre frío y en personas susceptibles como los ancianos. 3) Sin embargo, esta relación no parece detectarse (según la medida de los captadores de la ciudad elegidos en el estudio) con niveles de humos negros inferiores a $80-90 \mu \mathrm{g} / \mathrm{m}^{3}$ y sí a partir de estos valores, lo que sugeriría la existencia de un umbral a partir del cual el contaminante tendría efectos en la mortalidad.

\section{AGRADECIMIENTOS}

A Rosa Ruiz, del EUSTAT, y al Instituto Nacional de Meteorología por facilitar parte de los datos que fueron necesarios para la realización de este trabajo. A M. Jesús Iturritxa por su ayuda en el análisis de las muestras de contaminantes. Y a los componentes de los grupos EMECAM de Barcelona y Valencia, en especial a Ferrán Ballester, por el estímulo y las sugerencias aportadas para llevar a cabo este proyecto. 
Figuras 7 y 8

Gráficos de dispersión y ajuste no paramétrico de regresiones localmente ponderadas (lowess) de la relación entre los residuos ajustados de mortalidad y el primer retardo de humos negros. Vitoria-Gasteiz, 1990-94
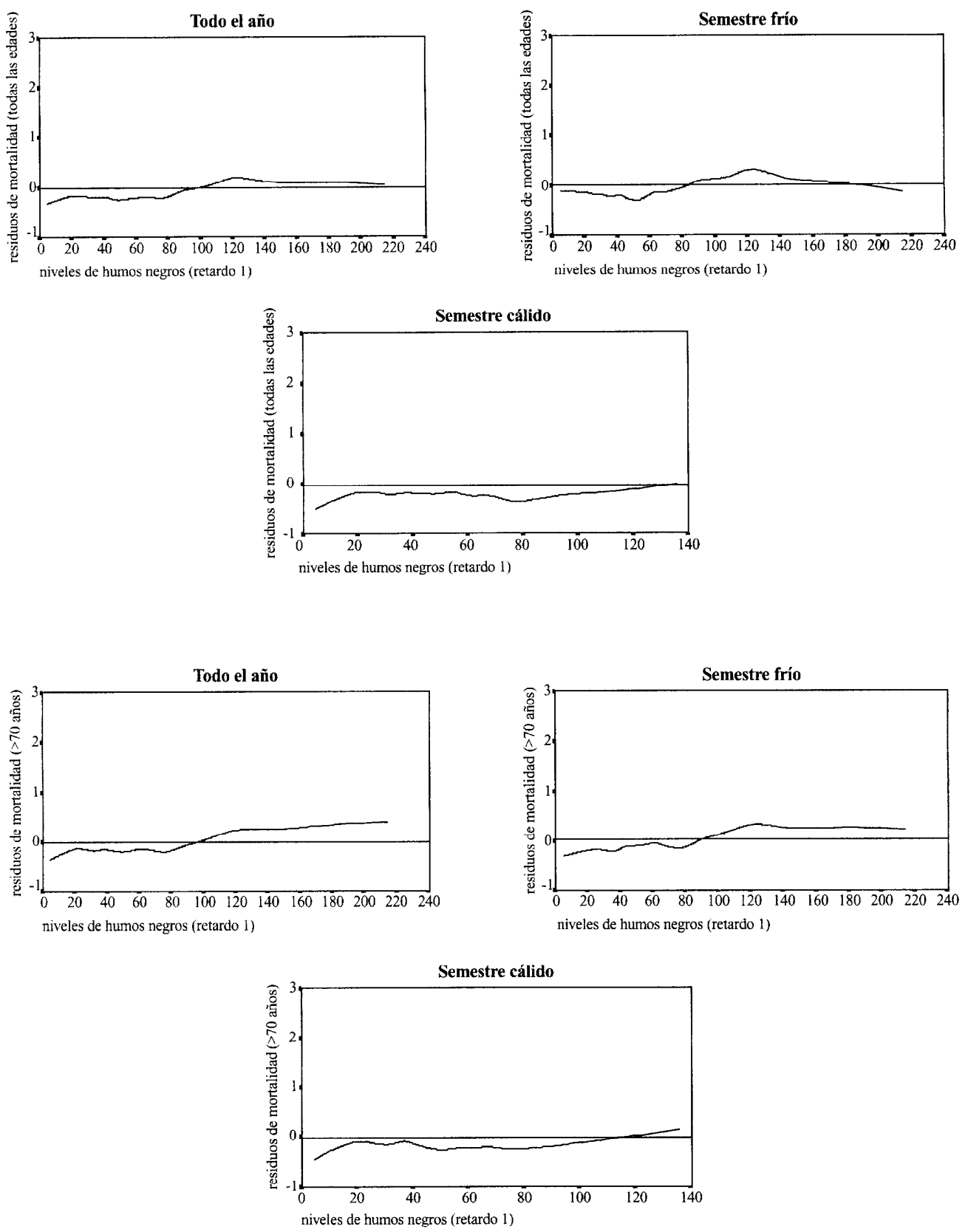


\section{BIBLIOGRAFÍA}

1. Derrienic F, Richardson S, Mollie A, Lellouch J, Short term effects of sulphur dioxide pollution on mortality in two French cities. Int J Epidemiol 1989; 18: 186-97.

2. Verhoeff AP, Hoek G, Schwartz J, Van Wijnen $\mathrm{JH}$. Air pollution and daily mortality in Amsterdam. Epidemiol 1996; 7: 225-30.

3. Touloumi G, Samoli E, Katsouyanni K. Daily mortality and "winter type" air pollution in Athens Greece - a time series analysis within the APHIEA project. J Epidemiol Community Health 1996; 50(1 Suppl): 47S-51S.

4. Zmirou D, Barumandzadeh T, Balducci F, Ritter P, Laham G, Ghilardi JP. Short term effects of air pollution on mortality in the city of Lyon, France, 1985-90. J Epidemiol Community Health 1996; 50 (1 Suppl): 30S-35S

5. Wojtyniak B, Piekarski T. Short term effect of air pollution on mortality in Polish urban populations-what is different? J Epidemiol Community Health 1996; 50 (1 Suppl): 36S-41S.

6. Spix C, Wichmann HE. Daily mortality in Köln. J Epidemiol Community Health 1996; 50 (1 Suppl): 52S-58S.

7. Sunyer J, Castellsagué J, Sáez M, Tobias A, Antó JM. Air pollution and mortality in Barcelona. $J$ Epidemiol Community Health 1996; 50 (1 Suppl): $76 \mathrm{~S}-80 \mathrm{~S}$.
8. Ballester F, Corella D, Pérez-Hoyos S, Hervás A. Air pollution and mortality in Valencia, Spain: a study using the APHEA methodology. J Epidemiol Community Health 1996; 50: 527-33.

9. Hoek G, Schwartz JD, Groot B, Eilers P. Effects of ambient particulate matter and ozone on daily mortality in Rotterdam, The Nederlands. Arch Environ Health 1997; 52:455-63.

10. Anderson HR, Ponce de León A, Bland JM, Bower JS, Strachan DP. Air pollution and daily mortality in London: 1987-92. BMJ 1996; 312:665-9.

11. Pérez-Hoyos S, Sáez M, Barceló MA, Cambra K, Figueiras A, Ordoñez IM et al. Protocolo EMECAM: análisis del efecto a corto plazo de la contaminación atmosférica sobre la mortalidad. Rev Esp Salud Publica 1999; 73:177-185.

12. Ballester F, Sáez M, Alonso E, Taracido M, Ordoñez JM, Aguinaga I, et al. El proyecto EMECAM. Estudio multicéntrico español sobre la relación entre la contaminación atmosférica y la mortalidad. Antecedentes, participantes, objetivos, material y métodos. Rev Esp Salud Pública $1999 ; 73: 165-175$.

13. Katsouyanni K, Touloumi G, Spix C, Schwartz J, Balducci F, Medina S et al. Short term effects of ambient sulphur dioxide and particulate matter on mortality in 12 European cities: results from time series data from the APHEA project. BMJ 1997; $314: 1658-63$.

14. Committee of the environmental and occupational health assembly of the American Thoracic Society. Health effects of outdoor air pollution, Am J Respir Crit Care Med 1996; 153; 3-50. 